\title{
Intraday Asymmetric Test of the CSI300 Index Futures Based On eGARCH Model
}

\author{
Junbo Wang a, Susheng Wang, Guanglu Li
}

School of economics and management, Harbin Institute of Technology, Shenzhen, China

a407995864@qq.com

Keywords: High frequency data, eGARCH model, CSI 300 index futures, intraday volatility

\begin{abstract}
In this paper, the internal high frequency samples of stock index futures are taken as the research object, and the statistical characteristics of the high frequency data in the stock index futures are studied. After determining the stable data, the eGARCH model is used to test the asymmetry of the volatility in the stock index futures. The model parameter estimation results show that all the selected three samples have asymmetry, and the impact of the conditional variance of stock index futures is very strong. The futures market has been greatly shocked, and it will be difficult to disappear in the short term.
\end{abstract}

\section{Introduction}

Volatility generally refers to a disturbance that deviates from the mean value with time and is a measure of the uncertainty and risk of the underlying assets. Therefore, volatility is generally used to measure the volatility of the underlying assets or the rate of return on investment. In 1982, Engle [1] proposed autoregressive conditional heteroscedasticity (ARCH) model, which provides a framework for the research of volatility. Bollerslev (1986) [2] modifies the ARCH type, adding the moving average term of conditional heteroscedasticity in $\mathrm{ARCH}$, and puts forward the GARCH type. In order to study the asymmetry of volatility, Nelson (1991) [3] proposed an eGARCH model. Gao Wei (2015) [4] uses GARCH model to study the overnight interest rate volatility of financial market Shibor. It is found that there is a significant asymmetric effect in the distribution of Shibor overnight sequence under the GARCH family model fitting. Besides the EGARCH model, the GARCH family model can eliminate the ARCH effect of financial product sequence better. In the past research, volatility is generally asymmetric. Therefore, we use eGARCH model to study the asymmetry of intraday volatility in Shanghai and Shenzhen 300 stock index futures.

\section{Methodology}

In order to reflect the asymmetry of financial market volatility, Nelson (1991) [1] proposed an exponential GARCH (eGARCH model). The model can be expressed as:

$$
\begin{gathered}
a_{t}=\sigma_{t} \varepsilon_{t} \\
\sigma_{t}^{2}=\alpha_{0}+\sum_{i=1}^{m} \alpha_{i} a_{t-i}^{2}+\sum_{j=1}^{s} \beta_{j} \sigma_{t-j}^{2} \\
g\left(\varepsilon_{t}\right)=\theta \varepsilon_{t}+\gamma\left[\left|\varepsilon_{t}\right|-E\left(\left|\varepsilon_{t}\right|\right)\right]
\end{gathered}
$$

Where, $\left\{\alpha_{i}\right\}, \mathrm{i}=1,2, \cdots \mathrm{m},\left\{\beta_{j}\right\}, \mathrm{j}=1,2, \cdots \mathrm{s}$. are model coefficient, Function $\mathrm{g}(\cdot)$ satisfied $\mathrm{E}_{t-1}\left(\mathrm{~g}\left(\varepsilon_{t}\right)\right)=0$.The use of a piecewise function can be expressed as:

$$
g\left(\varepsilon_{t}\right)= \begin{cases}(\theta+\gamma) \varepsilon_{t}-\gamma E\left(\left|\varepsilon_{t}\right|\right), & \varepsilon_{t} \geq 0 \\ (\theta-\gamma) \varepsilon_{t}-\gamma E\left(\left|\varepsilon_{t}\right|\right), & \varepsilon_{t}<0\end{cases}
$$

In the formula, when $\gamma \neq 0$, indicates that the interference on the impact of asset prices is asymmetric, when the $\gamma<0$ shows the influence of financial assets by the negative external shocks than by the external impact, this is called $\gamma$ leverage effect.

\section{Empirical}

The object of this study is the intraday high frequency volatility of Shanghai and Shenzhen 300 stock index futures. Therefore, this paper selects the high frequency intraday high frequency data of 
Shanghai and Shenzhen 300 stock index futures as the research samples. For the sake of generality, three high-frequency data samples of trading days were selected as the research objects. The stock index futures trading data of two consecutive samples by the CSI 300 stock index futures contract IF1207 and IF1208 from July 2, 2012 to July 20123 and February 19, 2013. In order to deal with the same transaction at the same time at the same time, a continuous futures contract is constructed according to the most active volume of the transaction volume and the month. The reason for this selection is to take into account the continuity of the stock index futures trading day in July, and the holiday effect has the least effect on the intraday volatility. All the data come from the China Financial Futures Exchange. Suppose $\mathrm{p}_{\mathrm{t}}$ is the CSI 300 index futures price at time $\mathrm{t}$, the return fluctuation can be defined as follows:

$$
\mathrm{r}_{\mathrm{t}}=100 *\left(\ln \mathrm{p}_{\mathrm{t}}-\ln \mathrm{p}_{\mathrm{t}-1}\right)
$$

Table 1.The statistical characteristics of the CSI 300 index futures return fluctuation

\begin{tabular}{ccccccccc}
\hline Sample & Number & Average & Std.Err & Min & Max & Skewness & Kurtosis & S.t \\
\hline Sample1 & 29568 & 0.000 & 0.010 & -0.060 & 0.060 & 0.010 & 1.310 & 0.000 \\
Sample2 & 30087 & 0.000 & 0.010 & -0.060 & 0.060 & 0.040 & 1.900 & 0.000 \\
Sample6 & 31337 & 0.000 & 0.010 & -0.060 & 0.100 & 0.080 & 2.750 & 0.000 \\
\hline
\end{tabular}

Remark: The result is the data of return Multiply 100.

It can be seen that the Shanghai and Shenzhen 300 stock index futures intraday high frequency data sampling days observation sample number of about 32400, data preprocessing within days after the effective observation samples at around 30000, indicating that the effective price of observation samples recorded high rate, high liquidity, require higher sampling frequency to record price information. It is found that data preprocessing has no effect on the mean and variance of the observed samples. The mean value is zero and the variance is 0.01 . The influence of sample skewness and kurtosis pretreatment is large, and the skewness and kurtosis of the observed samples decrease obviously. We focus the research on the observed samples. It can be seen from the sample statistics that although the skewness of the processed samples is small, most of them are positive, and the distribution of their returns is not completely symmetrical, which may reflect the leverage of highfrequency financial assets, which needs further verification. The kurtosis of the observed samples is less than 3, which indicates that the observed samples do not have the peak tailing, and we think it is related to the sampling frequency. The following picture shows the distribution characteristics of the daily return on the Shanghai and Shenzhen 300 stock index futures:



Figure 1. The fluctuation of CSI 300 index futures return 
From the over charts, we can see that the Shanghai and Shenzhen 300 stock index futures intraday return rate fluctuates basically symmetrical, and the volatility range of high frequency intraday volatility is narrow, but the abnormal value is more. There is no obvious intraday characteristics, but there is a certain volatility aggregation phenomenon. This shows that the Shanghai and Shenzhen 300 stock index futures have a very strong liquidity and a greater risk.

Due to the high order GARCH model leads to excessive GARCH and ARCH coefficients, the model is unstable. Therefore, we establish the eGARCH $(1,1)$ model to fit the residual model. The following table is the best result of the GARCH model which is established by using the rugarch package in the $\mathrm{R}$ language:

Table 2. The optimal estimation of eGARCH model parameters

\begin{tabular}{|c|c|c|c|c|c|c|}
\hline GARCH model & Parameters & Value & Error & T test & $\operatorname{Pr}(>|\mathbf{t}|)$ & Significance \\
\hline & $m u$ & -0.003 & 0.002 & -1.527 & 0.127 & - \\
\hline & mal & -0.110 & 0.058 & -1.889 & 0.059 & . \\
\hline Fit11 & omega & 0.001 & 0.005 & 0.295 & 0.768 & - \\
\hline \multirow[t]{5}{*}{ Sample1(eGARCH) } & alphal & -0.097 & 0.027 & -3.548 & 0.000 & $* * *$ \\
\hline & betal & 1.000 & 0.000 & 3436.566 & 0.000 & $* * *$ \\
\hline & gammal & 0.089 & 0.020 & 4.457 & 0.000 & $* * *$ \\
\hline & $m u$ & 0.000 & 0.003 & -0.066 & 0.947 & - \\
\hline & mal & 0.020 & 0.063 & 0.310 & 0.756 & - \\
\hline Fit12 & omega & -0.629 & 0.200 & -3.145 & 0.002 & $*$ \\
\hline \multirow[t]{5}{*}{ Sample2(eGARCH) } & alphal & 0.069 & 0.040 & 1.749 & 0.080 & $\cdot$ \\
\hline & betal & 0.898 & 0.032 & 27.991 & 0.000 & $* * *$ \\
\hline & gammal & 0.149 & 0.067 & 2.232 & 0.026 & $*$ \\
\hline & $m u$ & -0.004 & 0.003 & -1.128 & 0.259 & - \\
\hline & mal & -0.120 & 0.063 & -1.901 & 0.057 & . \\
\hline Fit13 & omega & -0.470 & 0.514 & -0.914 & 0.361 & - \\
\hline \multirow[t]{3}{*}{ Sample3(eGARCH) } & alphal & -0.076 & 0.043 & -1.745 & 0.081 & . \\
\hline & betal & 0.913 & 0.094 & 9.672 & 0.000 & $* * *$ \\
\hline & gammal & 0.275 & 0.124 & 2.216 & 0.027 & $*$ \\
\hline
\end{tabular}

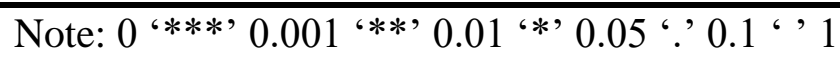

According to the coefficient estimation results of the eGARCH model, the coefficient garmmy is not zero, which indicates that the Shanghai and Shenzhen 300 stock index futures have a strong asymmetry. The estimation of model coefficients shows that the values of the coefficients and the values of the model variance equations are all less than 1 , and the alpha + beta $<1$, which indicates that the eGARCH models established are stable convergent. The sum of alpha and beta is very close to 1 , indicating that the impact of the conditional variance of stock index futures is very strong. The futures market has been greatly shocked, and it will be difficult to disappear in the short term. The volatility of the impact will be sustained in a certain period of time. 


\section{Conclusion}

In this paper, the eGARCH model is used to test the asymmetry of HF 300 stock index futures in the day of high frequency return. The results show that:

1). The CSI 300 stock index futures have a strong asymmetry;

2). The eGARCH model can describe the dynamic volatility process of stock index futures in a better way.

3). The impact of the conditional variance of stock index futures is very strong.

\section{References}

[1]. Engle, R.F. Autoregressive Conditional Heteroscedasticity with Estimates of the Variance of United Kingdom Infl. in Econometrica. 1982.

[2]. Bollerslev, T., Generalized autoregressive conditional heteroskedasticity. Eeri Research Paper, 1986. 31(3): p. 307-327.

[3]. Nelson, D.B., Conditional Heteroskedasticity in Asset Returns: A New Approach. Modelling Stock Market Volatility, 1991. 59(2): p. 347-370.

[4]. Gao Wei, statistical research on volatility of China's Shibor financial market based on GARCH model, statistics and decision, 2015 (10): page 30-33. 